\title{
Dédoublement du genre et opérations de fictionnalisation : faire " comme si c'était vrai », ou divers visages de l'altération didactique
}

Genre duplication and fictionalisation operations: Doing " as if it were true ", or the different faces of didactic alteration

Yann Vuillet, Jean-Paul Mabillard, Catherine Tobola Couchepin et Joaquim Dolz

\section{OpenEdition \\ Journals}

Édition électronique

URL : https://journals.openedition.org/educationdidactique/1482

DOI : 10.4000/educationdidactique.1482

ISSN : 2111-4838

Éditeur

Presses universitaires de Rennes

\section{Édition imprimée}

Date de publication : 30 octobre 2012

Pagination : 89-102

ISBN : 978-2-7535-1984-8

ISSN : $1956-3485$

\section{Référence électronique}

Yann Vuillet, Jean-Paul Mabillard, Catherine Tobola Couchepin et Joaquim Dolz, « Dédoublement du genre et opérations de fictionnalisation : faire « comme si c'était vrai », ou divers visages de l'altération didactique », Éducation et didactique [En ligne], 6-2 I octobre 2012, mis en ligne le 30 octobre 2014, consulté le 25 avril 2022. URL : http://journals.openedition.org/educationdidactique/1482 ; DOI : https://doi.org/10.4000/educationdidactique.1482 


\title{
DÉDOUBLEMENT DU GENRE ET OPÉRATIONS DE FICTIONNALISATION :
}

\author{
FAIRE « COMME SI C'ÉTAIT VRAI ", \\ OU DIVERS VISAGES DE L'ALTÉRATION DIDACTIQUE \\ Yann Vuillet (Haute École Pédogogique du Valais) \\ Jean-Paul Mabillard (Haute École Pédogogique du Valais) \\ Catherine Tobola Couchepin (Haute École Pédogogique du Valais) \\ Joaquim Dolz (FPSE, Université de Genève)
}

\begin{abstract}
Résumé : Cette contribution convoque, en les approfondissant, les concepts de dédoublement générique impliqué par la scolarisation de genres textuels (Schneuwly \& Dolz, 1997) ainsi que celui d'opérations de fictionnalisation - au sens de Scheuwly $(1988,2003)$ et de Bernié (1998) - pour décrire certaines difficultés d'enseignement et d'apprentissage particulières. En se livrant à une analyse fine de la notion de destinataire dans un contexte de transposition didactique d'un genre argumentatif, les auteurs mettent en évidence qu'une forme de fictionnalisation fictive témoigne de l'intrication des phénomènes de dédoublement du genre et de fictionnalisation en situation scolaire.
\end{abstract}

Mots clés : genre textuel, transposition didactique, dédoublement générique, fictionnalisation, difficultés d'enseignement et d'apprentissage

Yann Vuillet, Jean-Paul Mabillard, Catherine Tobola Couchepin \& Joaquim Dolz

Dans le cadre d'une recherche financée par un fond DORE ${ }^{1}$ - laquelle porte sur les capacités et les difficultés des apprenants en situation de production de texte, sur les conditions d'enseignement et d'apprentissage d'un texte argumentatif et sur les interactions entre l'enseignant et les élèves lors du déroulement d'une séquence d'enseignement - nous avons l'occasion de mettre en évidence certaines difficultés d'enseignement et d'apprentissage relativement singulières.

Ces difficultés, observables au sein de productions écrites d'élèves d'une même classe aussi bien qu'à travers les interactions orales ayant eu lieu lors de la mise en situation opérée par l'enseignant, résultent globalement de la transposition didactique d'un aspect commun à toutes les situations d'activités langagières complexes, et qui s'est vu conceptualisé par Schneuwly $(1988,2003)$ et Bernié (1998) en terme de fictionnalisation. Plus exactement, c'est le traitement des opérations de fictionnalisation impliquées par le dédoublement générique (Schneuwly \& Dolz, 1997) découlant de la scolarisation de la « réponse au courrier des lecteurs » $(\mathrm{RCL})$ qui va retenir notre attention. À cet égard, il conviendra avant tout de différencier scrupuleusement ce que nous entendons par fiction- nalisation de ce que nous analyserons en tant que dimension génératrice de fiction de la scolarisation d'un genre textuel. En effet, la terminologie existante peut, ici, entraîner certains quiproquos nuisibles à la clarté de l'analyse.

Afin d'analyser comment les opérations de fictionnalisation et l'effet générateur de fiction du genre scolarisé entrent en résonance et marquent les apprentissages, nous analyserons les interactions entre enseignant et élèves au cours de modules d'enseignement ainsi que les productions initiales et finales de ces derniers. Cet article se propose donc de définir dans un premier temps les rapports entretenus entre le dédoublement du genre et les opérations de fictionnalisation, avant de les exploiter pour mieux comprendre les interactions didactiques dans la classe ainsi que leurs effets sur les productions des élèves.

De notre point de vue, la notion de fictionnalisation et les opérations qui vont avec, traitées ici du point de vue de la didactique du français, permettent d'aborder des situations d'enseignement d'autres didactiques disciplinaires. Notre contribution pourra ainsi fournir des bases pour un regard comparatiste dans la conclusion de cet article. 
Dédoublement du genre et opérations de fictionnalisation: des concepts pour analyser des situations didactiques et leurs produits

\section{Du genre scolarisé comme générateur de fiction}

Pour permettre aux élèves de maîtriser diverses pratiques langagières, l'école convoque des pratiques sociales de référence et les apprête sous la forme de situations didactiques. Les enseignants créent ou recourent ainsi généralement à des situations de communication fictives qui ont pour but d'ancrer les apprentissages de leurs élèves dans un semblant de réalité. Si toute production écrite à l'école peut ainsi être assimilée à de la fiction, il n'en demeure évidemment pas moins que les spécificités de ces situations de communication sont bien réelles, et dignes d'intérêt.

Cet ancrage particulier nécessite donc que soient précisés les rapports que le cadre scolaire institue (ou insinue) entre des genres de textes authentiques et leurs versions fictives. À la suite de Schneuwly et Dolz (1997), nous pouvons ainsi admettre que, comme tout genre de texte qui se trouve décontextualisé hors des pratiques sociales de référence et recontextualisé en situation scolaire, la RCL subit une forme de dédoublement. En effet, ce genre ne relève alors plus seulement d'un outil de la communication, mais devient également objet d'enseignement et d'apprentissage (et ce, que la tâche scolaire implique un destinataire existant en dehors de l'école aussi bien qu'un destinataire imaginaire). Ce dédoublement implique qu'élèves et enseignants se retrouvent « [...] dans un espace du comme si, où le genre fonde une pratique langagière qui est nécessairement en partie fictive puisqu'elle est instaurée à des fins d'apprentissage. » (Schneuwly \& Dolz, 1997, p. 30).

En ouvrant un espace du comme si, les genres scolarisés agissent en somme à la manière de générateurs de fiction. De la fiction est effectivement générée à l'endroit symbolique où l'authenticité des situations de communication de référence rencontre l'artificialité des situations d'enseignement et d'apprentissage. Les situations scolaires s'écartent forcément de celles vécues hors de l'école: par conséquent, si l'on se risque à employer ici le terme d' "artificialité », c'est bien pour pointer le caractère de (re)construction culturelle de la situation de communication scolarisée, et non pour l'évaluer en termes de «faux » ou de «falsifié ». Deux pôles mettent donc en tension l'espace didactique du comme si: en lui coïncident un lien et une rupture. Un lien, en ceci qu'il permet l'acquisition de savoirs relatifs à une pratique sociale de référence; et une rupture, en cela que l'espace du comme si n'est ni celui du quotidien connu, ni celui des pratiques langagières de référence. En somme, pour se rapprocher d'un genre de référence, l'école le transforme forcément et s'en éloigne donc d'autant. Or, puisque la génération d'une fiction est tout simplement inhérente à la scolarisation même d'un genre, l'école n'a d'autre choix que de s'accommoder de la rupture paradoxale qui l'accompagne - bien que cette dernière puisse opposer certaines difficultés (nous le verrons) à l'apprentissage de savoirs relatifs à un genre donné.

Nous savons déjà que les situations de communication scolaires générées par la didactisation d'un genre - tout aussi bien adaptées aux caractéristiques des conduites langagières visées qu'à leur enseignement - contribuent globalement à donner du sens aux apprentissages, mais il demeure du ressort de la recherche de préciser le rôle de ces dernières dans le développement des capacités langagières des élèves.

\section{De la fictionnalisation}

Maintenant esquissées certaines dimensions paradoxales des genres scolarisés pris comme des générateurs de fiction, il nous faut développer (succinctement) un aspect que la RCL partage avec de nombreuses pratiques langagières, et qui peut poser problème lors de leur enseignement et de leur apprentissage. Cet aspect, conceptualisé par Schneuwly (1988, 2003) et Bernié (1998) en terme de fictionnalisation, va également nous servir à analyser et à comprendre par quoi, et comment est orienté l'enseignement du genre qui nous concerne ici.

Le concept de fictionnalisation renvoie au fait que toute situation langagière complexe suppose la capacité, pour l'énonciateur comme pour le destinataire, de reconstruire et de se représenter les paramètres du contexte de production (voire, de réception). Ce concept met ainsi en évidence que « les paramètres du contexte (énonciateur, destinataire, but et lieu social) ne sont que partiellement les instances physiques et sociales de la production et de la réception immédiates 
et doivent être fictionnalisées pour fonctionner comme base d'orientation de la production [...] » (Schneuwly, 2003). En d'autres termes, l'énonciateur (mais aussi son destinataire) se représente ou reconstruit forcément le contexte d'énonciation - qu'il soit authentique ou fictif - pour pourvoir la production discursive d'une base d'orientation. Suivant Bernié (1998, p. 169), les traces de cette reconstruction par l'énonciateur sont en outre "visibles dans le discours » qu'elles « structurent à travers diverses médiations »: c'est en effet sur cette qualité de la fictionnalisation que nous allons sous peu nous appuyer pour formuler quelques observations. Nous allons ainsi mettre en évidence que la représentation du (double) contexte de communication marque les interactions en classe, certains gestes didactiques de l'enseignant (en particulier ceux de régulation), certains obstacles rencontrés par les élèves (voir Gagnon 2010) ainsi que, finalement, les productions des élèves.

S'agissant d'observer et d'analyser un enseignement portant sur un genre de texte tel que la RCL, le concept de fictionnalisation apparaît doublement utile. Il permet d'une part de penser la reconstruction et la représentation des paramètres du contexte impliqués par la pratique sociale de référence. Il permet d'autre part de rendre compte de ce que, en classe, les interactants doivent nécessairement reconstruire et se représenter ce qui oriente les activités - soit, en l'occurrence, la maîtrise d'une situation de communication de référence (la $\mathrm{RCL}$ ), laquelle est modalisée à travers un dispositif la rendant partiellement fictive. En d'autres termes, le dédoublement du genre entraînant le dédoublement des opérations de fictionnalisation, l'enseignant et les élèves se retrouvent face à deux espaces de fictionnalisation partiellement distincts: partiellement seulement, car bien que l'un (véhiculé par la pratique sociale de référence) et l'autre (impliqué par la scolarisation du genre) ne coïncident pas absolument, ils s'offrent néanmoins généralement en une seule et même fois, sous une seule et même forme - celle de la situation de communication de l'activité scolaire. On comprend donc d'autant mieux qu'effectuer une opération de fictionnalisation à partir d'un genre scolarisé puisse constituer une difficulté bien réelle pour l'enseignement comme pour les apprentissages.

Les interactions observées au sein d'une classe vont nous permettre d'avancer qu'une logique dyna- mique et circulaire peut s'établir entre les différents espaces de fictionnalisation et l'effet de générateur de fiction du genre scolarisé. Nous allons mettre en évidence que les processus de fictionnalisation développés en classe à partir de la fiction initiale proposée par les moyens d'enseignement peuvent profondément transformer les données de cette dernière.

\section{Du simulacre à la simulation}

Avant cela, précisons que la nécessité didactique de faire comme si l'activité langagière se réalisait pour de vrai, suivant ses modes de présentation en classe, peut renvoyer à des logiques très différentes. Puisque la scolarisation d'un genre entraîne nécessairement sa sémiotisation (ou, pour le dire avec un néologisme, son en-signement) l'intérêt est grand d'observer quel statut peut, en classe, être accordé à cette « mise en signes ». La façon dont le dispositif didactique se voit lui-même pris en considération (ou non) comme paramètre du contexte d'énonciation de l'activité scolaire peut en effet affecter profondément la base d'orientation de la production d'un genre scolarisé. Différents effets peuvent être observés au sein des échanges en classe, comme au sein des productions des élèves. En prenant la précaution de préciser $a$ priori quelles peuvent être ces logiques et ces façons, nous nous apprêtons donc à nous munir d'autant d'outils pour nos analyses à venir ${ }^{2}$.

En premier lieu, la scolarisation d'un genre peut se traduire par une situation de communication fictive tendant au simulacre (au sens d'une apparence qui se donne pour une réalité). Dans ce cas, la dimension fictive du dispositif d'enseignement et d'apprentissage n'est pas donnée comme telle par l'enseignant, et reste donc généralement ignorée des élèves. Le dispositif véhicule ou est véhiculé par une prétention à la "réalité », à la vérité référentielle - autrement dit, il se revendique ou est revendiqué en tant que pratique sociale de référence, en tant que situation de communication «authentique». Il convient en outre de différencier cette prétention à la vérité référentielle selon qu'elle est implicite - auquel cas aucun commentaire n'est formulé pour assurer de la (fausse) réalité de la situation de communication -, ou au contraire explicite, et prenant donc la forme d'un discours ayant pour but d'affirmer la réalité de la situation de communication. 


\section{DÉDOUBLEMENT DU GENRE ET OPÉRATIONS DE FICTIONNALISATION}

Yann Vuillet \& al

D'une autre manière, par opposition au simulacre, une situation de communication fictive peut explicitement renvoyer au fonctionnement sémiotique d'une simulation. Dans ce cas, la dimension fictive des activités d'enseignement et d'apprentissage tend à être assumée comme telle par l'enseignant, et connue des élèves. La raison de la présence à l'école d'une telle activité ne tient pas dans le fait qu'elle soit en ellemême une pratique sociale de référence, mais bien qu'elle y ressemble et qu'elle peut y conduire Cette ressemblance sera donc toujours explicitée à travers une consigne, un commentaire ou un discours.

Tels que nous les entendons, le simulacre et la simulation sont à considérer en tant que pôles (et non en tant qu'états) orientant l'organisation d'une situation d'enseignement. Cette opposition ne saurait donc suffire à rendre totalement compte de la multiplicité et de la grande plasticité de ces situations complexes.

\section{De la fictionnalisation passée à travers un générateur de fiction: Jean et ses avatars}

Pour travailler la RCL, les moyens d'enseignement officiels suisses romands « S'exprimer en français » (2001) proposent aux enseignants de progresser en différentes étapes. La première, dite de la « mise en situation », ne s'appuie sur aucun matériel fourni par les moyens: à travers des discussions sur un ou plusieurs thèmes, elle doit permettre aux élèves de mieux cerner une situation d'argumentation, de se représenter le projet d'écriture et de s'approprier ainsi des contenus susceptibles d'être réutilisés lors des activités. La deuxième étape doit amener les élèves à rédiger une première production faisant, en principe, l'objet d'une évaluation par l'enseignant. L'étape de la production initiale s'appuie sur différents exercices proposés par les moyens, et en particulier sur la lettre suivante:

Je m'adresse au courrier des lecteurs car j'ai un problème. J'en ai assez que maman m'accompagne tous les jours en voiture à l'école. Je veux y aller à vélo. Or, mes parents me l'interdisent. Ai-je raison ou pas? Si j'ai raison, comment les convaincre?

Merci pour vos futures réponses.
Afin d'identifier, à partir de cette lettre, quels savoirs relatifs aux situations de communication pourraient faire l'objet d'un enseignement, nous voulons en proposer une analyse a priori en nous appuyant sur les catégories utilisées par Bronckart (1996) pour définir les conditions de production des textes. Ce dernier désigne ainsi, avec la situation d'action langagière, les « mondes formels (physique, social et subjectif) qui sont susceptibles d'exercer une influence sur les productions textuelles » (p. 93). Suivant l'auteur, puisque l'« agent» (p. 44) est en quelque sorte le dépositaire de certaines versions de ces mondes formels (lesquels renvoient à des ensembles de représentations sociales), il conviendrait encore de différencier la situation d'action langagière dans son acception externe - telle qu'une « communauté d'observateurs pourrait (la) décrire »-de son acception interne ou effective soit, telle qu'un « agent les a intériorisées » (p. 94) ${ }^{3}$. En situation d'action langagière, la mobilisation par un agent de certaines de ses « représentations des mondes» (Bronckart, 1996, p. 95) est simultanément orientée dans deux directions: ces représentations déterminent aussi bien le(s) contenu(s) thématiques (ou référents) du texte que le contexte de la production textuelle. Alors que le contexte de la production textuelle peut, toujours suivant Bronckart, se voir abordé sous l'angle de ses aspects physiques de même que sous celui de ses aspects sociaux et subjectifs, notre souhait d'effectuer une analyse a priori des savoirs enseignables relatifs aux situations de communication impliquées par un enseignement portant sur la RCL suppose que l'on dédouble nos analyses. En effet, suivant la logique de dédoublement du genre scolarisé, c'est bel et bien sur l'intrication de deux situations de communications que nous nous pencherons par la suite.

Suivant cette perspective, notre analyse a priori prend donc la forme de deux tableaux, lesquels mettent chacun en évidence: 1) le contexte de production impliqué par la pratique sociale de référence du genre RCL - ce que nous faisons en tenant compte du cas particulier de la controverse qui nous occupe; 2) la transposition de ce contexte de production, en classe, en objet d'enseignement. Le premier des tableaux (fig. 1) concerne les aspects physiques des contextes de production, tandis que le second (fig. 2) - qui nous intéresse davantage - renvoie aux aspects sociaux et subjectifs des situations d'interaction sociale. 
1) Pratique sociale de référence de la réponse au courrier des lecteurs

Lieu matériel de production

Moment de production

Locuteur

Récepteur

$$
\text { lieu L (variable) }
$$

temps T (variable)

la personne quiproduit physiquement la lettre
2) Réponse au courrier des lecteurs transposée en classe

$$
\text { une salle de l'école }
$$

90 minutes d'une matinée les enfants no. de la classe (en tant que personnes)

les personnes concrètes susceptibles de l'enseignant N. (en tant que personne) lire la lettre

Fig. 1: Aspects physiques des contextes de production

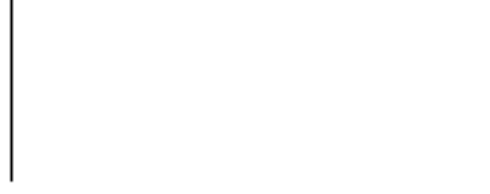

Lieu social

Position sociale de l'énonciateur

Position sociale du (des) destinataire(s)

But

\section{1) Pratique sociale de référence de la réponse} au courrier des lecteurs

rubrique d'un courrier des lecteurs d'une publication pour enfants

lecteur du journal pour enfants, au bénéfice de différents rôles et positions potentiels.

Jean en tant qu'enfant qui vit une controverse avec ses parents; les parents de Jean en tant que vivant une controverse avec leur enfant; le responsable de la rubrique ; les lecteurs du journal

donner son avis quant à savoir si Jean a raison de vouloir aller à l'école à vélo ; si oui, l'aider à convaincre ses parents

\section{2) Réponse au courrier des lecteurs} transposée en classe

classe de français de la $4^{e}$ primaire élève de $4^{e}$ primaire enseignant apprendre à rédiger des textes argumentatifs à travers le genre de la réponse au courrier des lecteurs

Fig. 2: Aspects sociaux et subjectifs des situations d'interaction sociale 


\section{DÉDOUBLEMENT DU GENRE ET OPÉRATIONS DE FICTIONNALISATION}

Yann Vuillet \& al

De cette analyse a priori, il ressort que les savoirs relatifs aux situations de communication suivants pourraient faire l'objet d'un enseignement: au niveau du lieu social de la situation d'interaction, l'enseignant peut mettre en évidence que la RCL s'inscrit dans la rubrique d'un courrier des lecteurs d'une publication pour enfants; au niveau de la position sociale de l'énonciateur, les élèves peuvent apprendre qu'ils écrivent avant tout en tant que lecteurs du journal pour enfants (au bénéfice, certes, de différents rôles, de différentes positions sociales potentielles); pour ce qui est de la position sociale du (des) destinataires, des savoirs relatifs à la multiplicité potentielle de ce(s) dernier(s) (Jean, ses parents, le responsable de la rubrique, les lecteurs de la rubrique, etc.) sont susceptibles de se voir enseignés; finalement, un enseignement peut encore porter sur le but poursuivi par la réponse à la lettre de Jean, à savoir de donner son avis sur l'objet de la controverse, voire, d'aider Jean à convaincre ses parents.

Notre analyse a priori apparaît également utile pour comprendre que ce qui se joue en classe à travers cette situation de communication fictive consiste, pour l'élève, à écrire à la fois dans une rubrique au courrier des lecteurs et dans une classe, à écrire tout en même temps en tant que lecteur de cette rubrique et en tant qu'élève de $4^{\mathrm{e}}$ primaire, à s'adresser à tous les destinataires potentiels (et fictifs) de sa lettre ainsi que, simultanément, à son enseignant, mais encore à ajouter au but impliqué par la pratique sociale de référence celui d'un apprentissage... Libre à l'enseignant de présenter la situation de communication ainsi que la figure du destinataire (Jean) entre les pôles du simulacre et de la simulation, sachant que les deux peuvent coexister. Certes, formulée ainsi, la tâche semble extrêmement compliquée; on pourrait même être tenté, à la lecture de ces tableaux, de chercher un moyen pour simplifier le tout. Or il s'agit, ici, de garder à l'esprit que cette complexité est intrinsèque à toute situation d'enseignement et d'apprentissage: quoi que l'on y fasse, la scolarisation d'un genre impliquera toujours le dédoublement des aspects sociaux et subjectifs de la situation d'interaction.

\section{Démarche d'analyse}

Dans cet article, nous nous proposons d'explorer une démarche méthodologique nous permettant d'observer comment le dédoublement du genre et les opérations de fictionnalisation se déploient effectivement au cours de l'enseignement. Pour ce faire, nous avons tout d'abord enregistré et transcrit l'ensemble d'une séquence didactique réalisée dans une classe de $4^{e}$ primaire et portant sur la RCL. Le support utilisé par l'enseignant est la séquence didactique de la série COROME (Dolz, Noverraz \& Schneuwly, 2001) dont nous venons d'effectuer une analyse a priori en fonction de ses contenus - relatifs à la situation de communication - enseignables. Nous avons par ailleurs recueilli les textes des productions initiales et finales des élèves.

Grâce à la collecte de ces diverses données, nous avons pu: a) analyser les productions initiales et finales; b) établir le synopsis et la macrostructure de la séquence d'enseignement selon les techniques du GRAFE (Schneuwly \& Dolz, 2009); c) analyser les interactions verbales entre l'enseignant et les élèves des extraits portant sur la situation de communication dans un module d'enseignement, et ce, en vue de dégager la didactisation des dimensions contextuelles du genre de référence (cf. fig. 2) - plus spécifiquement le(s) destinataire(s) du courrier des lecteurs.

Le destinataire des productions initiales et finales des élèves nous étant apparu doté d'une identité pour le moins complexe et problématique, une analyse qualitative des formes d'implication de ce dernier dans les textes s'est avérée nécessaire. La comparaison entre les productions initiales et finales permettra donc, dans cette perspective, d'observer les transformations résultant de l'enseignement. Celles-ci sont formulées à partir d'une lecture croisée des productions écrites des élèves, des interactions orales ayant eu lieu en classe, et des synopsis des deux premières étapes de la séquence.

L'analyse des interactions didactiques, pour sa part, se focalise sur les interventions locales des enseignants et des élèves portant sur les éléments pointés, plus haut, dans notre analyse a priori des savoirs enseignables relatifs à la situation de communication de la RCL (cf. fig. 2). Le processus de prise en considération de l'inéluctable dédoublement fictif de la situation de communication lors de l'enseignement et de l'apprentissage d'un genre textuel est vu, ici, comme un geste professionnel caractéristique de 
la didactisation de la production écrite, et dont les modalités de réalisation méritent d'être décrites.

\section{Analyses et résultats}

Pour faire suite à notre analyse a priori et à la présentation de notre démarche méthodologique, nous allons désormais présenter nos observations portant sur les deux premiers modules de la séquence. Nos analyses montreront que l'activité de fictionnalisation impliquée par la production d'un texte et la dimension génératrice de fiction du genre scolarisé entrent en résonance d'une manière relativement complexe, puisqu'elles donnent en somme lieu à une forme de "fictionnalisation fictive». De même, il apparaitra que cette «fictionnalisation fictive ", parce qu'elle conduit les élèves et l'enseignant à reconstruire d'une manière très particulière le destinataire premier de la RCL (Jean), entraîne la non-prise en considération de nombreux savoirs relatifs aux situations de communication qui ont pourtant étés discutés et mis en évidence par l'enseignant durant la séquence d'enseignement. De la sorte, nous allons enfin pouvoir établir que, paradoxalement, les stratégies déployées par l'enseignant dans le but de faciliter à ses élèves la fictionnalisation de la situation d'interaction sociale fictive offrent aux élèves une aide qui se transformera ensuite en obstacle potentiel quant à la maîtrise du genre de référence.

\section{Mise en situation d'un genre dédoublé}

À l'occasion de la mise en situation, après avoir interrogé les élèves sur les manières par lesquelles ils se rendent eux-mêmes à l'école (à pied, en voiture, à vélo ?), l'enseignant décide d'anticiper le module de la production initiale et d'introduire le personnage de Jean. Dès les premiers instants, le statut de ce dernier (est-il réel ou fictif?) aussi bien que la raison de son apparition dans le cadre de la classe (a-t-il besoin d'aide, est-il le prétexte à un apprentissage?) apparaissent relativement problématiques:

E: [...] on a un problème avec JEAN // lis-voir ce qu'il y a d'écrit au tableau Z.

Élève Z.: les parents de Jean lui interdisent d'aller à l'école à vélo
E: ah/donc on n'a pas j'ai pas pris quelqu'un de la classe vous voyez car on a bien discuté un petit moment là alors maintenant on imagine qu'on a un copain dans cette classe qui s'appelle jean et ce jean eh bien il a écrit une lettre dans un journal/justement à l'endroit où on a le droit d'écrire des choses/vous avez déjà vu ça dans une revue que vous lisez/un endroit où c'est les lecteurs qui écrivent quelque chose

Une fois l'inscription au tableau noir (« Les parents de Jean lui interdisent d'aller à l'école à vélo ») lue par un élève, avant même de convoquer les connaissances que ses élèves ont du genre social de référence dont la maîtrise est visée (ce qu'il fera par la suite en se référant, par exemple, à un numéro du magazine «J'aime lire » apporté en classe par un élève), le premier élément que l'enseignant estime devoir préciser concerne l'identité problématique de ce Jean. L'enseignant assume en son nom le choix d'un enfant qui ne vient pas de la classe ( « donc on n'a pas j'ai pas pris quelqu'un de la classe vous voyez $[\ldots] »)$. Ce faisant, il anticipe - en l'explicitant partiellement - la situation de communication qui orientera l'activité articulée au genre scolarisé: ses élèves écriront à une personne qui n'est pas dans leur classe. Cependant, l'identité extrascolaire de Jean se trouve aussitôt contrebalancée par l'enseignant - sans doute pour permettre à ses élèves de " présentifier » ce correspondant imaginaire ${ }^{4}$ : " alors maintenant on imagine qu'on a un copain dans cette classe qui s'appelle Jean et ce Jean eh bien il a écrit une lettre dans un journal ». Sous l'impulsion de l'enseignant, et par le pouvoir d'imagination de ses élèves, Jean est ainsi destitué de son récent simulacre d'existence extrascolaire et fictivement situé dans la classe. En d'autres termes, peut-être pour atténuer le paradoxe qui consiste à écrire réellement à un personnage imaginaire, l'enseignant assure indirectement les élèves de la classe de l'existence du correspondant avant de leur demander d'imaginer que ce dernier est l'un des leurs. Les indications données par l'enseignant pour persuader ses élèves que Jean est une personne authentique, et non un personnage fictif, passent également par l'authentification de sa lettre comme ayant été réellement écrite:

E. : les parents de Jean lui interdisent d'aller à l'école à vélo moi je vous ai juste mis le thème là tout à l'heure on verra je vous distribuerai une fiche où (il remontre un petit carré sur une page de magazine)on verra son 


\section{DÉDOUBLEMENT DU GENRE ET OPÉRATIONS DE FICTIONNALISATION}

Yann Vuillet \& al

petit texte vraiment tel que lui l'a écrit d'accord ce sera pas long vous verrez il a écrit dans un journal son problème alors dites moi maintenant juste avant qu'on commence (il se tourne vers le texte inscrit au tableau) pensez-vous qu'ils ont raison pourquoi si oui pourquoi si non pourquoi et puis que conseillez-vous à Jean/alors la discussion est lancée

De la sorte, les élèves devraient, aux dires de l'enseignant, découvrir un texte réellement écrit par Jean. Ces premières observations indiquent que l'enseignant semble choisir spontanément la voie du simulacre pour préparer ses élèves à l'opération de fictionnalisation de la situation de communication (fictive) induite par la séquence didactique. Ce faisant, il parvient dans un premier temps à éluder la dimension génératrice de fiction du genre scolarisé, et à « naturaliser » ce dernier. Cependant, les choix de l'enseignant n'apparaissent plus aussi nettement dirigés lorsqu'il présente enfin le texte que Jean est, jusque-là, supposé avoir rédigé:

E : VOILA notre première fiche de travail/cette fiche c'est presque comme si on avait photocopié une page de journal justement où on a le courrier des lecteurs on va la découvrir ensemble (toussotement) c'est simplement le début $[. .$.

D'un moment à l'autre, les élèves de la classe passent ainsi du registre d'un « petit texte vraiment tel que lui (Jean) l'a écrit » à celui du «c'est presque comme si on avait photocopié une page de journal »: effectivement, la lettre de Jean n'a pas tout à fait été photocopiée d'une page de journal. Bien que «S'exprimer en français " recoure à des reproductions de lettres réellement parues dans des revues pour enfants, celle de Jean s'est évidemment trouvée transposée dans des moyens d'enseignement, sélectionnée par l'enseignant (transpositions externes), puis apprêtée in situ par ce dernier lors de la mise en situation ayant lieu en classe avant de finalement parvenir aux élèves (transpositions internes). Peu à peu, alors que l'on vient de passer de la demande de Jean à la copie de celle-ci, il apparaît que l'enseignant renonce progressivement à la réalité du correspondant pour amener ses élèves à le considérer comme quelqu'un qu' « on ne connaît pas du tout », pas plus que l'on ne connaît le lieu à partir duquel il écrit:
E. : [...] ]si nous on veut répondre à Jean dans la revue parce que c'est quelqu'un qu'on connait pas du tout qui habite n'importe où en Suisse en Europe comment je dois faire alors $\uparrow$ (il désigne un élève de la tête)

Élève $\mathrm{y}$ : ben: XXX

E. : $\mathrm{mm}$

Élève y: et puis ben heu: des fois je crois qu'il y a l'adresse be heu on fait la lettre puis ensuite on l'envoie à l'adresse E. : d'accord t'as raison on va voir sur la page du j'aime lire il y a une adresse et on envoie la réponse on écrit (geste avec la main) comme une/petite carte une petite lettre et puis on l'envoie donc NOTRE REPONSE

Jean est-il réel? Imaginaire? Les élèves doiventils faire comme s'il était assis en classe à côté d'une élève, ou alors comme s'il était un habitant parfaitement inconnu écrivant depuis la Suisse, l'Europe? À l'image du genre dédoublé par sa scolarisation - dédoublement impliquant celui de l'opération de fictionnalisation des contextes de production et de réception épistolaires -, le Jean présenté par l'enseignant a une identité multiple: tant et si bien qu'une fois venu le moment d'évoquer l'activité de production d'une lettre en réponse au courrier de ce personnage, on assiste à l'échange suivant:

\section{$\mathrm{E}:[\ldots]$ ça se sera à NOUS}

Élève $\mathrm{y}$ : mais pour de faux

E: ben non pas pour de faux

Élève $\mathrm{x}$ : il va la recevoir $\uparrow$

E : il va pas la recevoir $\uparrow$ si t'entends ça par pour de faux oui pour de faux mais on va écrire une réponse à Jean

Élève $S$ : mais heu $\mathrm{H}$

E : d'accord $\uparrow$

Élève $\mathbf{x}$ : on va la mettre dans j'aime lire.

$\mathrm{E}$ : on va écrire une réponse à Jean ben non parce que c'est pas $\mathrm{H}$ il dit c'est pour de faux oui dans le sens là c'est pour de faux c'est pas: Jean il a peut-être écrit 
je sais pas peut-être qu'il a écrit ça dans une revue mais c'est pas le pour écrire ça donc nous ON VA lui répondre D'ACCORD Z. $\uparrow$ MOTIVE $\uparrow$ bon c'est bien mais c'est comme si c'était vrai après ben c'est justement pour s'entrainer une fois et si une fois vous voulez répondre à quelqu'un dans une vraie revue ceux qui ont des revues à la maison ben vous saurez le faire mais évidemment que là on sait pas si c'est vrai ou pas c'est pas une vraie revue c'est un exercice d'accord A. $\uparrow$

\section{OUI X个(il désigne un élève de la tête)}

Élève $\mathbf{x}$ : mais heu mais c'est vrai que Jean il a ça $\uparrow$

E: que Jean il a écrit cette lettre $\uparrow c ' e s t$ justement ce qu'a dit A. on en sait rien mais c'est pas important de savoir si c'est vrai ou pas mais peut-être qu'ils ont pris les gens qui ont fait les fiches ils ont peut-être pris des vraies lettres dans un courrier comme si moi j'avais pris ici une vraie lettre d'enfant hein $\uparrow$ sûrement ou ils ont peut-être inventé j'en sais rien oui (il désigne un élève de la tête)

Élève y: (seul ?)

E: TOUT SEUL comme des grands ouais comme si t'étais à la maison dans ton salon VOILA vous avez les devoirs qui sont prêts pour demain/bon appétit

À travers les interrogations (on en aurait à moins) que les élèves soulèvent au sujet de ce Jean qui ne cesse de devenir autre, pas une seule fois son existence n'est remise en question. Pour les élèves, en revanche, il importe de savoir s'ils écriront vrai- ment à ce correspondant, s'il recevra leurs lettres, ou si ses parents lui interdisent réellement d'aller à l'école à vélo. À la fin de cette première leçon, il est frappant de constater que ce sont les élèves qui contraignent ainsi l'enseignant à lever le voile sur la dimension fictive de la situation de communication, et à exposer du même-coup les rouages de la « machinerie » sémiotico-didactique qui oriente les apprentissages. Bien que «les gens qui ont fait les fiches ils ont peut être pris des vraies lettres dans un courrier ", le simulacre se brise aussitôt le dispositif didactique révélé comme tel. Le changement de statut sémiotique de la situation de communication fictive inscrit finalement cette dernière dans l'ordre de la simulation: on écrit à l'école pour « s'entrainer », pour « une fois [...] répondre à quelqu'un dans une vraie revue».

\section{Registre sémiotique et fictionnalisation: mouvement général et effets sur les productions des élèves}

En prenant en considération l'intrication existant entre le dédoublement du genre et le dédoublement des opérations de fictionnalisation, l'analyse des extraits présentés met en évidence que les interactions suivent un mouvement général guidé par les diverses identités successivement prêtées à Jean, lequel modifie également la valeur sémiotique de la situation de communication didactique proposée. Ce double mouvement, simplifié, peut être schématisé comme suit:

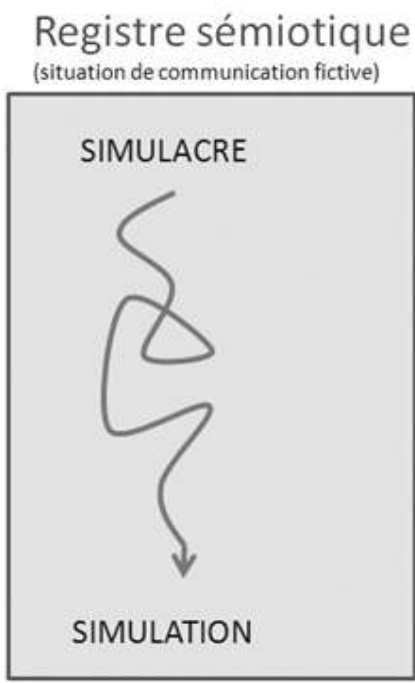

Jean et ses avatars

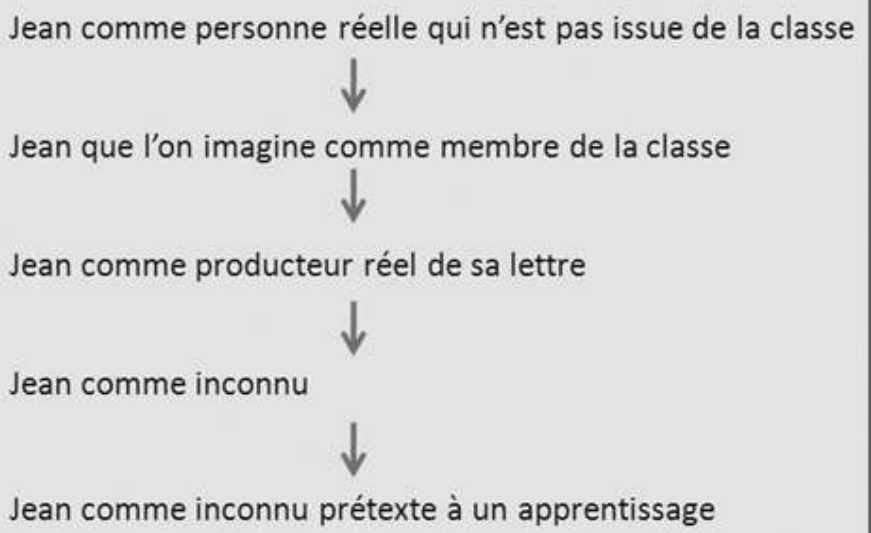




\section{DÉDOUbLEMENT DU GENRE ET OPÉRATIONS DE FICTIONNALISATION}

Yann Vuillet \& al

À force d'enchaînements, de retours en arrière, de superpositions ou encore de ruptures, il apparaît ainsi que l'évolution de l'identité de Jean transforme la situation de communication fictive et la fait passer de l'ordre du simulacre à celui de la simulation (laquelle est donc explicitement mise en relation avec une visée d'apprentissage). Ceci étant, nous pouvons maintenant nous intéresser aux effets que ce traitement de la fictionnalisation a pu avoir dans les productions des élèves. Pour l'exemple, nous allons nous baser sur les productions initiale et finale de l'un d'entre eux. Il s'agit d'observer ici, dans un premier temps, quel est le Jean à qui l'élève s'adresse, mais aussi d'interroger, en retour, quel est l'élève qui s'adresse à ce Jean. Nous pourrons ensuite déduire de ces productions quelles aides et quels obstacles ont été suscités par les interventions de l'enseignant.

Je m'adresse au courrier des lecteurs car j'ai un problème. J'en ai assez que maman m'accompagne tous les jours en voiture à l'école. Je veux y aller à vélo. Or, mes parents me l'interdisent. Ai-je raison ou pas? Si j'aí raison, comment les convaincre?

Merci pour vos futures réponses.

Jean

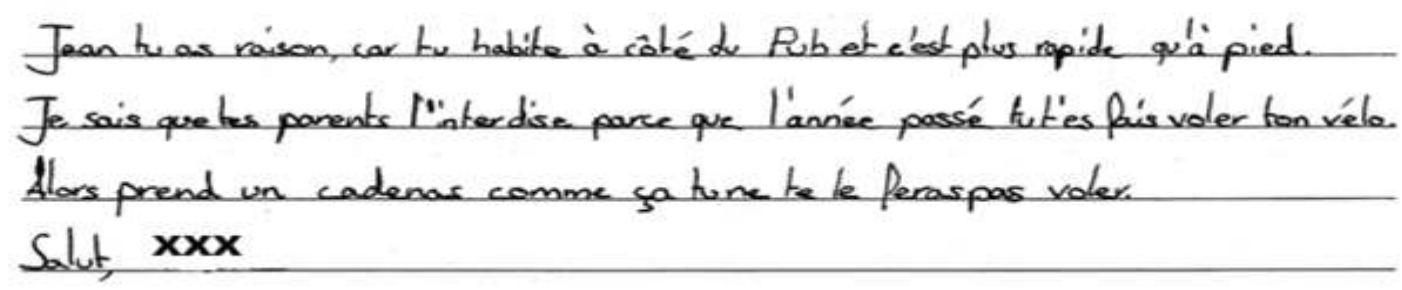

Production initiale de l'élève $X$

Je m'adresse au courrier des lecteurs car j'ai un problème. J'en ai assez que maman m'accompagne tous les jours en voiture à l'école. Je veux y aller à vélo. Or, mes parents me l'interdisent. Ai-je raison ou pas? Si j'ai raison, comment les convaincre?

Merci pour vos futures réponses.

Jean

Cher Jean,

Je pence que tes parents ont vraiment tort de t'empếcher d'ater à l'écle à vélb.

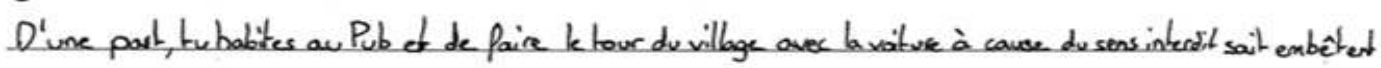

D'aute part, Il paut en profiter car à Champóry, th' ya pas beawcoup de circulation.

Ensuite, personne lement j'aime bien pouvoir $m$ 'aérer ks poumons.

Mais surtout, tu as qu'a venir avec moi au Bike Park j'ivais des fisen été, je peuxte dire ceet super

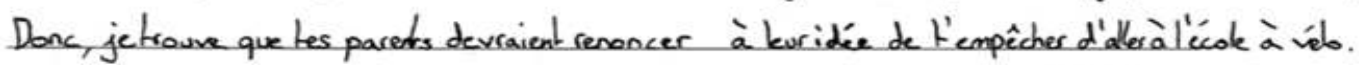


À la lecture de ces deux productions, nous pouvons constater que le Jean auquel s'adresse l'élève en question n'est de toute évidence pas celui qui, à l'origine, a réellement écrit une lettre au courrier des lecteurs d'une revue. De même, ces deux productions n'ont pas non plus pour destinataire le Jean dont l'existence est suggérée par les moyens « S'exprimer en français ». En fait, il apparaît que le producteur de ces deux lettres s'adresse en fin de compte à l'un des Jean dont l'identité s'est progressivement vue façonnée en classe: ce Jean qui habiterait le même village (de montagne) que les élèves, qui aurait sensiblement le même âge qu'eux, et qui pourrait fort bien, somme toute, être l'un de leurs amis. Nous pouvons ainsi constater que les conditions de validité de 4 des 5 arguments apparaissant dans ces deux productions dépendent d'un Jean qui serait un alter ego de l'élève.

Plus encore, de la production initiale à la production finale, l'élève développe sensiblement les détails de l'existence fictive de Jean pour étayer ses propres arguments; il n'est par exemple plus seulement mentionné que Jean habite «à côté du Pub» (1), mais aussi que la rue correspondant à cette adresse est un sens interdit (2): le renforcement de la représentation de la vie fictive de Jean conduit ainsi directement à renforcer la pertinence de l'argument. De la sorte, il devient évident que le choix fait par l'enseignant de demander à ses élèves d'imaginer qu'ils connaissent Jean s'avère une aide lorsqu'il s'agit, pour eux, d'inventer des arguments: s'adresser à une personne connue est en effet moins exigeant, en termes de gestion discursive, que d'écrire à une personne dont on ignore tout. Néanmoins, en l'état, les arguments produits ne sont pas plus généraux que généralisables (aspect commun à nombre d'arguments de qualité, bien qu'il ne fasse pas explicitement partie des attentes des moyens d'enseignement utilisés pour des élèves de ce degré); la personnification de Jean, ce socle sur lequel s'appuie la stratégie argumentative, constitue donc également une pierre d'achoppement pour la maîtrise visée du genre de référence - lequel suppose la méconnaissance du destinataire principal de la RCL, ainsi que la prise en considération de la multiplicité silencieuse des destinataires potentiels de celle-ci.

Là ne se limitent cependant pas les effets observables de la « reconstruction » de l'identité du destinataire fictif sur les deux productions en question. À travers ces dernières, l'auteur laisse encore entendre qu'il connaît personnellement le destinataire - il est au courant de certaines de ses mésaventures (comme le vol de son vélo une année auparavant)-, si bien qu'il va jusqu'à l'inviter à venir faire du vélo en sa compagnie: c'est dire combien l'identification proposée par l'enseignant lors de sa mise en situation joue à plein pour l'élève! Ce dernier constat nous mène plus loin: en s'adressant à un personnage fictif, en lui inventant une vie, en s'imaginant un passé et un futur commun avec lui, c'est sa propre existence que l'auteur de ces deux lettres place dans l'espace du comme si. En d'autres termes, sous l'effet de la force "génératrice de fiction » du genre scolarisé et par l'intermédiaire des opérations de fictionnalisation que celui-ci implique, l'élève s'inscrit dans une situation d'énonciation hautement complexe qui entraîne sa propre fictionnalisation en tant qu'énonciateur.

\section{Conclusion}

En pratiquant une analyse croisée des interactions verbales ayant eu lieu lors de la mise en situation, des productions des élèves et du synopsis de la séquence, nous sommes parvenus à mettre en évidence plusieurs phénomènes d'un intérêt certain quant à l'enseignement et à l'apprentissage de la production écrite d'un genre textuel tel que la RCL. Nous constatons ainsi, en toute logique, que le dédoulement subit par un genre textuel lors de sa scolarisation entraîne, pour l'enseignant comme pour les élèves, le dédoublement des opérations de fictionnalisation. L'intrication entre ces opérations et l'effet « générateur de fiction » dû au dédoublement du genre conduit ainsi à une forme de « fictionnalisation fictive » dont le statut sémiotique peut varier, allant des formes scolaires du simulacre à celles de la simulation.

À cet égard, il apparaît que les stratégies développées par l'enseignant ont des effets ambivalents - et durables - sur les productions des élèves. Elles répercutent le paradoxe voulant qu'un genre scolarisé, vis-à-vis des pratiques langagières de référence, relève autant du lien que de la rupture. En effet, parmi toutes les identités de Jean apparaissant successivement en classe, si les élèves se réfèrent principalement à un destinataire qui habite leur village c'est bien que l'enseignant est parvenu à faire un lien avec certains 


\section{DÉDOUBLEMENT DU GENRE ET OPÉRATIONS DE FICTIONNALISATION}

Yann Vuillet \& al

aspects du quotidien connu de ses élèves. Mais ce rapprochement, néanmoins, se paie au prix d'une certaine mise à distance des paramètres du contexte d'énonciation orientant, dans les pratiques langagières de référence, la production d'une RCL. Les lettres des élèves ne s'adressent en fait ni au Jean du courrier des lecteurs, ni à celui des moyens d'enseignement, mais bien à cet avatar fictif qui a pris forme en classe. Bien que l'ancrage géographique de ce Jean (en montagne, tout comme les jeunes Suisses ayant participé à l'expérience) aide visiblement les élèves à inventer des arguments, ces derniers ne jouissent en revanche que rarement d'une valeur « générale » - ce qui appauvrit d'autant les argumentaires développés. De la même manière, on peut remarquer que le lieu social de production des lettres est bien celui de l'école, et non celui d'une rubrique de RCL. Encore une fois, notre réflexion ne vise aucunement à relativiser l'intérêt pour l'apprentissage qu'offrent les diverses situations d'enseignement impliquant le dédoublement du genre scolarisé et celui des opérations de fictionnalisation: ces phénomènes apparaissent indissociables de la transposition didactique d'un genre textuel donné. Nos analyses nous conduisent finalement à proposer quelques principes de fonctionnement didactique associés d'une part à la recontextualisation, dans un cadre scolaire, de pratiques langagières de référence, et d'autre part aux modalités de distanciation-rapprochement résultant des opérations de fictionnalisation, et conduisant à des transformations voire à l'effacement de certains paramètres de la situation de communication de référence. Ces principes interdépendants sont les suivants:

a. Principe d'ubiquité du dédoublement du genre: la scolarisation d'un genre implique que l'élève réponde à deux exigences distinctes et simultanées, s'inscrivant en même temps dans deux lieux sociaux disjoints (mais dans un seul lieu physique).

b. Principe d'éloignement dû au rapprochement: la recontextualisation du genre de texte, la personnalisation du destinataire et la focalisation sur ce dernier sont des gestes développés par l'enseignant dans un but de médiation et d'adaptation aux élèves, et orientent les productions de ces derniers. Pour donner du sens comme pour motiver les élèves, l'enseignant tend à rapprocher certains éléments fictifs orientant leurs produc- tions (le destinataire, son lieu de vie) de leurs réalités quotidiennes. La captatio par rapprochement rend cependant plus difficile le traitement des situations de communications exigeant une certaine prise de distance. La prise en considération de l'altérité que constituent les élèves de la classe a donc pour effet collatéral l'altération de la pratique langagière de référence.

c. Principe d'u-topos des genres scolarisés: sous l'effet conjoint des deux principes précédents, le développement de savoirs scripturaux par l'intermédiaire de la fiction éloigne le produit final d'un destinataire plus abstrait et complexe, tel qu'impliqué par la RCL, ainsi que d'un lieu social tangible, établi dans la pratique sociale de référence. En ceci, les lieux relatifs construits à l'école, bien qu'ils n'existent nulle part ailleurs sous cette forme précise, sont des u-topies nécessaires à l'enseignement.

Ces trois principes ont en commun leurs qualités paradoxales: à travers eux, c'est une même logique d'altération (au sens de "rendre autre pour un autre ») inhérente à l'enseignement de la textualité qui se laisse observer. Ses différentes modalités méritent d'être prises en considération dans l'analyse des gestes des enseignants et des apprentissages des élèves, d'autant plus que cette logique d'altération affecte également l'identité discursive même que l'élève développe dans sa production scolaire. Intégrée aux pratiques d'écriture, comme nous le voyons dans les productions analysées, cette logique fait de l'élève l'énonciateur d'une forme de fiction scolaire où luimême se met en scène dans une relation imaginaire.

Il conviendrait peut-être, désormais, d'observer et d'analyser quel(s) rôle(s) les situations d'énonciation renvoyant à une forme de fiction scolaire tiennent, ou pourraient tenir dans les activités devant mener à la maîtrise d'un genre textuel de référence donné.

Nous émettons par ailleurs l'hypothèse que la notion de fictionnalisation, introduite en didactique du français par Schneuwly (1988) et développée par Bernié (1998), n'est aucunement exclusive des situations de production écrite, et qu'elle intervient aussi dans d'autres situations scolaires impliquant un énonciateur et un destinataire. On pourra tout d'abord l'exploiter dans les différentes sous-disci- 
plines de l'enseignement du français langue première et langue étrangère. De ce point de vue, les situations d'enseignement de l'oral pourraient procéder selon nos hypothèses (qui restent à vérifier) à partir des mêmes opérations. De la même manière, concernant les approches didactiques de la littérature, certains phénomènes relatifs à la construction scolaire de la figure d'auteur ou d'énonciateur dans les textes gagneraient certainement à se voir approchés par l'entrée de la fictionnalisation.

La théorie des genres et sa transposition à l'enseignement fournissent un cadre nous permettant de voir, d'une manière plus fine, certains processus de fictionnalisation associés à différentes situations de communication, qui ne concernent d'ailleurs pas uniquement les diverses sous-disciplines du français, mais bien l'ensemble de la didactique des langues. Au-delà de la didactique des langues (et à l'intérieur de celles-ci pour aborder l'enseignement grammatical), toute tâche scolaire contextualisée peut impliquer les opérations de fictionnalisation. Ce sera par exemple le cas des problèmes d'arithmétique dans lesquels interviennent un Pierre, un Paul ou un Jean, mais ce sera également le cas des matchs d'entraînement opposants les joueurs d'une même équipe.

Les régularités et les spécificités disciplinaires, à partir de la notion de fictionnalisation introduite en didactique du français, restent à étudier: nous ne voulons nous risquer ici à une généralisation excessive de leur utilité. Reste que nous sommes convaincus de l'apport potentiel que cette notion peut représenter. Il nous semble surtout important, pour un chercheur en didactique disciplinaire, de connaître les stratégies des enseignants pour aider les élèves à donner du sens aux situations formelles d'apprentissage dans ce qu'elles ont de fictif. L'ambigüité des effets sur la relation aux pratiques sociales de référence est à notre avis constitutive du didactique: l'étude empirique des continuités et des ruptures entre les situations extérieures à l'école et celles des situations d'apprentissage demeure ainsi fondamentale pour toutes les didactiques disciplinaires. 


\section{DÉDOUBLEMENT DU GENRE ET OPÉRATIONS DE FICTIONNALISATION}

Yann Vuillet \& al

\section{NOTES}

1. Cette recherche a été financée par le fond DORE n. 13DPD3-120167.

2. À noter que nous ne prenons ici en considération que les situations d'enseignement/apprentissage où l'activité de production de texte est inscrite dans une situation de communication fictive: une situation de communication authentique pourrait être traitée d'une manière similaire, mais non identique.

3. Bien que nous souscrivions à la position de Bronckart, pour qui le chercheur n'a pas d'accès direct aux représentations internes dont un agent dispose de la situation d'action langagière (laquelle comprend, pêle-mêle, les diverses représentations que l'agent se fait de lui-même, du thème qu'aborde sa production ou encore du cadre communicatif qui la comprend), nous estimons néanmoins qu'en approchant une production langagière d'une manière compréhensive, il demeure possible de collecter certaines traces indirectes de ces représentations - ce qui nous renvoie, d'ailleurs, aux considérations de Bernié quant à la visibilité dans le discours de la reconstruction, par l'énonciateur, des paramètres de la situation communication (1998, cf.2.0.).

4. Plus tard dans la leçon, dans le même registre et non sans humour, l'enseignant va même plus loin: « maintenant on se branche sur Jean on l'imagine il est là il est assis à côté de F. hein Jean ».

\section{RÉFÉRENCES}

Bernié, J.-P. (1998). Éléments théoriques pour une didactique interactionniste de la langue maternelle. In M. Brossard \& J. Fijalkow (dir.) Apprendre à l'école, perspectives piagetiennes et vygotskiennes (p. 155-197). Talence: Presses Universitaires de Bordeaux.

Bronckart, J.-P. (1996). Activités langagières, textes et discours. Pour un interactionnisme socio-discursif. Neuchâtel et Paris: Delachaux et Niestlé.

Dolz, J., Noverraz, M. \& Schneuwly, B., (2001). S'exprimer en français. Séquences didactiques pour l'oral et pour l'écrit. Bruxelles, De Boeck.

Gagnon, R. (2010). Former à enseigner l'argumentation orale. De l'objet de formation à l'objet enseigné en classe de culture générale. Thèse n.452. FAPSE. Université de Genève.

Schneuwly, B. (1988). Le langage écrit chez l'enfant: la production des textes informatifs et argumentatifs, Paris, Delachaux \& Niestlé.

Schneuwly, B. (1997). Parole et fictionnalisation. Une voie pour l'enseignement de l'oral. Éléments pour une conférence donnée à la PUC-LAEL, São Paulo, Brésil

Schneuwly, B. (2003). Enseigner la parole publique: une approche sociohistorique. In B. Maurer (Éd.), Didactiques de l'oral (p. 58-67). Caen: CRDP de Basse-Normandie.

Schneuwly, B. \& Dolz, J. (1997). Les genres scolaires, des pratiques langagières aux objets d'enseignement. Repères, 15, p. 27-40.

Schneuwly, B. \& Dolz, J. (2009). Des objets enseignés en classe de français. Rennes, PUR. 